\title{
Demographic characteristics associated with awareness of cigarette health warnings and thinking about quitting among current adult cigarette smokers in Zambia, 2017
}

\author{
Masauso M. Phiri', April D. Summers², Alissa C. Kress², Isabel Garcia de Quevedo², Ralph Caraballo², Evelyn Twentyman²
}

\begin{abstract}
INTRODUCTION Noticing health warnings on cigarette packages has been associated with thinking about quitting. This study examined sociodemographic characteristics associated with awareness of health warnings on cigarette packages and thinking about quitting because of health warning labels among adults who currently smoked tobacco.

METHODS We analyzed data from the 2017 Zambia WHO STEPS survey (STEPwise approach to surveillance) for noncommunicable disease risk factors. Descriptive analyses and logistic regression were performed to assess the association of select sociodemographic characteristics with awareness of health warnings and thinking about quitting because of health warnings.

RESULTS Adults who currently smoked tobacco who were aged 30-44 years, of Chewa ethnicity, or with a formal education, were more likely to be aware of health warnings than those aged 18-29 years (adjusted prevalence ratio, APR $=1.26 ; 95 \%$ CI: $1.02-1.54)$, of Bemba ethnicity (APR $=1.43 ; 95 \%$ CI: $1.17-1.74)$, or with no formal education (APR: 2.61-5.95). Among all adults who currently smoked, those of Chewa ethnicity (APR=1.55; 95\% CI: 1.03-2.35), or with a formal education (APR:1.80-4.38), were more likely to report thinking about quitting because of health warnings than those who were of Bemba ethnicity or with less than primary school education level. Women who currently smoked were $49 \%$ less likely ( $\mathrm{APR}=0.51 ; 95 \% \mathrm{CI}$ : $0.23-0.84$ ) to report thinking about quitting than men. Among a subset of adults who currently smoked who were aware of health warning labels, no sociodemographic characteristics were significantly associated with thinking about quitting in unadjusted or adjusted models.

CONCLUSIONS Sociodemographic characteristics such as sex, ethnicity, and education level were significantly associated with awareness of cigarette health warnings. Among cigarette smokers aware of health warnings, no sociodemographic differences in thinking about quitting were found. Tobacco control campaigns may need to target people of ethnicities with the highest smoking prevalence in the country.
\end{abstract}

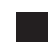

\section{AFFILIATION}

1 Tobacco Control Scholars Program Fellow Centre for Primary Care Research, School of Medicine, University of Zambia, Ridgeway, Zambia

2 Global Tobacco Control Branch, Office on Smoking and Health, National Center for Chronic Disease Prevention and Health Promotion, Centers for Disease Control and Prevention, Atlanta, United States

\section{CORRESPONDENCE TO}

Masauso M. Phiri. Tobacco Control Scholars Program Fellow Centre for Primary Care Research, School of Medicine, University of Zambia, Ridgeway Campus, Ridgeway, 10101 Lusaka, Zambia. E-mail: masauso@ unza.zm ORCID ID: https://orcid. org/0000-0001-7653-7988

\section{KEYWORDS}

sociodemographic characteristics, health warnings, cigarette, smoking, awareness, thinking about quitting

Received: 31 August 2021

Revised: 10 December 2021

Accepted: 12 December 2021

\section{INTRODUCTION}

Tobacco use is one of the leading causes of preventable disease, disability, and death ${ }^{1}$. While tobacco use is declining in high-income countries, it is increasing in certain low- and middle-income countries (LMICs) $)^{1,2}$.
In 2017, the prevalence of tobacco use and tobacco cigarette smoking in Zambia was $15.8 \%$ and $12.3 \%$, respectively ${ }^{3}$, and the most recent Demographic Health Surveys in Zambia suggest that the prevalence of tobacco use has been increasing especially among 
males, from $15 \%$ in $2000-2001$ to $19 \%$ in $2018^{4-7}$. In 2008, Zambia became a party to the World Health Organization Framework Convention on Tobacco Control (WHO FCTC) which provides evidencebased guidelines on how countries can implement and manage tobacco control measures ${ }^{8-10}$. The WHO FCTC outlines six proven measures toward tobacco demand reduction, including warning about the dangers of tobacco $^{11}$. Article 11 of the WHO FCTC requires the adoption of health warnings on tobacco packages with specific recommendations that the warnings be large (i.e. cover at least $50 \%$ and no less than $30 \%$ of the principal display areas), clear, and legible, and include both pictures and words to enhance their effectiveness ${ }^{8}$.

Health warning labels on cigarette packages serve as one of the primary means of communicating the harmful effects of cigarette smoking ${ }^{12,13}$. Awareness of the health harms of cigarette smoking has been associated with increased thinking about quitting and quit attempts among people who smoke ${ }^{13}$. Some countries in the African region such as Uganda and Mauritius are addressing the tobacco epidemic by strengthening implementation of the WHO FCTC; in particular, introducing pictorial health warnings covering more than $65 \%$ of tobacco product packaging ${ }^{14,15}$. Several studies that assessed the impact of health warnings concluded that pictorial health warnings were more effective than singletext warnings in achieving increased awareness and knowledge of tobacco harms, encouraging thinking about quitting among people who currently smoke, and preventing smoking initiation among people who do not smoke $\mathrm{i}^{13,16-22}$.

Although Zambia became a Party to the WHO FCTC in 2008, Zambia's policy on health warnings does not yet comply with WHO FCTC recommendations: current legislation fails to specify any minimum size of health warnings with respect to the cigarette package, and no pictures are required $^{2,23}$. A 2014 International Tobacco Control (ITC) country-wide survey found that only $69.0 \%$ of cigarette packages in Zambia had a visible text health warning in English, with the rest being either not visible or in another language ${ }^{23}$. In the 2017 WHO STEPwise approach to Surveillance (STEPS) survey, two-thirds $(66.9 \%)$ of adults who currently smoked tobacco who saw text-only health warning labels on cigarette packages in Zambia thought about quitting smoking, and the survey report did not delineate the factors that influenced such thinking about quitting ${ }^{3}$. Prior studies from high-income countries have suggested that the responses to health warnings vary based on many factors, including socioeconomic or sociodemographic factors such as sex, age, education level, and income levels ${ }^{24,25}$; however, there is a paucity of literature from LMICs in general ${ }^{26}$, and Zambia in particular. As has been stated in a number of studies ${ }^{25-27}$, there is a clear need for local data to support policy and in-country tobacco control strategies, especially in developing countries. The purpose of this study was, therefore, two-fold: 1) to examine sociodemographic characteristics associated with awareness of health warnings on cigarette packages among Zambian adults who currently smoke; and 2) to examine sociodemographic characteristics associated with thinking about quitting smoking because of the health warnings among adults who currently smoke.

\section{METHODS}

This study was a secondary analysis using data from the 2017 Zambia WHO STEPS survey. The aim of the survey was to establish a non-communicable disease (NCD) surveillance platform that collects baseline indicators on determinants of NCDs and their risk factors for policy and planning purposes. The survey integrated modified questions from the Tobacco Questions for Surveys (TQS), a subset of key questions from the Global Adult Tobacco Survey

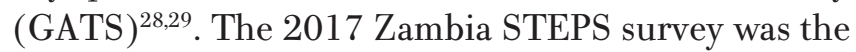
first nationally representative cross-sectional survey that collected comprehensive information on risk factors for NCDs, mental and oral health in adults aged 18-69 years in Zambia. A multi-stage cluster sampling technique was used to select a nationally representative sample of 5791 adults in Zambia, of which 4302 completed the survey, resulting in an overall response rate of $74.3 \%{ }^{3}$. More details on the sampling methods of the 2017 Zambia STEPS survey can be publicly accessed ${ }^{3}$. The University of Zambia Biomedical Research Ethics Committee (UNZABREC) approved the STEPS survey, and informed consent was obtained from participants ${ }^{3}$. Because deidentified secondary data were used, this study was considered exempt from CDC institutional review board approval. 


\section{Measures and variables}

The study population for this analysis included adults aged 18-69 years in Zambia who currently smoked tobacco. Adults who currently smoked tobacco were defined as those who answered 'Yes' to the question: 'Do you currently smoke any tobacco products, such as cigarettes, shisha, cigars or pipes?'. Two measures identified as key indicators in promoting thinking about quitting smoking ${ }^{16,18,19}$ were assessed in this study: 1) awareness of health warnings, and 2) thinking about quitting because of health warnings on cigarette packages. Health warning label awareness among adults who currently smoked tobacco was measured by the question: 'During the past 30 days, did you notice any health warnings on cigarette packages?'. The responses were 'Yes', 'No', 'Did not see any cigarette packages', or 'Don't know'. Responses other than 'Yes' were considered unaware of health warnings; these respondents were not questioned further regarding thinking about quitting smoking because of health warning labels as they were assumed to have no such intention per GATS/TQS guidelines ${ }^{28,29}$.

Respondents who indicated they were aware of health warnings were further asked about thinking about quitting using the question: 'During the past 30 days, have warning labels on cigarette packages led you to think about quitting?'. The responses were 'Yes', 'No' or 'Don't know'; 'Don't know' was categorized as 'No' for the purposes of this analysis. We calculated 2 measures of thinking about quitting smoking because of health warning labels; the first with a denominator of all adults who currently smoked, the second with a denominator of just those adults who currently smoked who were aware of health warnings. The numerator for both measures was the number of current smokers who thought about quitting smoking in the last 30 days because of the warning labels on cigarette packages. The first indicator assesses the prevalence of thinking about quitting smoking because of health warning labels among the total population of adult tobacco smokers in Zambia, consistent with GATS and TQS guidelines $^{28,29}$, while the second measure assesses thinking about quitting among the subset of smokers with awareness of the labels.

Sociodemographic variables included four age groups (18-29, 30-44, 45-59, 60-69 years), sex (male, female), ethnicity (Bemba, Chewa, Tonga, other), education level (no formal schooling, less than primary school, primary school completed, junior secondary school completed, secondary higher school completed or higher), marital status (currently married, not married), and annual household income $(\leq 10000,>10000$ and $\leq 20000$, $>20000 \mathrm{ZMW}$ ).

\section{Statistical analysis}

Descriptive analyses, including percentages and $95 \%$ confidence intervals (CIs) of awareness of health warnings and thinking about quitting were computed by sociodemographic characteristics. Chisquared tests were performed to test for significant differences in the distribution of demographic characteristics between those who were and were not aware of health warnings and those who did and did not intend to quit because of the health warnings; two-sided $\mathrm{p}<0.05$ was considered statistically significant. Logistic regression was performed to calculate crude (PR) and adjusted prevalence ratios (APR) for the association of sociodemographic characteristics with awareness of health warnings and thinking about quitting. Adjusted models included all sociodemographic characteristics of interest except for income, which was not included in the model due to a high proportion (approximately 24\%) of missing data. Results were suppressed if the unweighted denominator was $<25^{28,29}$. Analyses were conducted using SAS-callable SUDAAN version 11.0.

\section{RESULTS}

\section{Characteristics of the sample}

Overall, this study included a total of 475 adults who reported they currently smoked tobacco, which represents 916182 adults when survey weights were applied or $12.3 \%$ of adults in Zambia. Approximately $59.2 \%$ of tobacco smokers (unweighted $n=240$ ) reported awareness of cigarette health warning labels. Among all tobacco smokers, $37.4 \%$ (unweighted $\mathrm{n}=162$ ) reported thinking about quitting because of health warning labels; among those with awareness of the labels, $66.9 \%$ of respondents reported thinking about quitting because of the labels.

About three-quarters of all adults who currently smoked were aged $18-29$ years $(38.4 \%$; $95 \%$ CI: $32.2-44.9)$ and $30-44$ years $(36.6 \%$; $95 \%$ CI: 
31.4-42.0), and 91.7\% (95\% CI: 88.7-93.9) of all adults who currently smoked were male (Table 1 ). The highest proportion of adults who currently smoked had less than primary school education (28.9\%; 95\% CI: 24.2-34.2), followed by those who had completed primary school $(24.0 \%$; $95 \%$ CI: 19.6-29.1). Most were married (67.5\%; 95\% CI: 61.1-73.3) and close to half had earnings $\leq 10000$ ZMW (1000 Zambian Kwacha about US\$56) per annum (42.4\%; 95\% CI: 36.7-48.3). About onethird (34.0\%; 95\% CI: $27.3-41.5)$ of adults who currently smoked identified as Bemba while smaller proportions identified as Chewa and Tonga (8.1\%; 95\% CI: $5.3-12.2$, and $7.3 \%$; 95\% CI: 4.9-10.7, respectively); half identified as some other ethnicity (50.3\%; 95\% CI: 43.2-57.4).

\section{Distributions of sociodemographic characteristics by awareness of health warnings and thinking about quitting}

Among adults who currently smoked, significant differences in the distribution of sex, ethnicity, education level, and income were observed between those who were aware of health warning labels and those who were not (Table 1). Almost $95 \%$ of adult current smokers (94.6\%; 95\% CI: 91.1-96.7) who were aware of warning labels were male compared to 88.1\% (95\% CI: 83.2-91.7) among those who were not aware. More than one-third (35.6\%; 95\% CI: 26.6-45.9) of those who were aware were of Bemba ethnicity, $11.1 \%$ (95\% CI: 7.07-17.0) of Chewa ethnicity, and almost half (46.8\%; 95\% CI: 37.3-56.5) belonged to other ethnic groups while among those not aware 32.0\% (95\% CI: 24.2-40.9), 4.3\% (95\% CI: 1.9-9.3), and 54.9\% (95\% CI: 45.9-63.5) were of Bemba, Chewa, and other ethnicities, respectively. Most of those who were aware of health warnings had completed primary school (27.6\%; 95\% CI: 21.1-35.2) or junior secondary school (28.1\%; 95\% CI: $20.6-$ $37.2)$, and secondary or higher $(24.6 \%$; $95 \%$ CI: $18.3-$ $32.2)$ while, among those who were not aware, less than $40 \%$ had completed primary school (19.4\%; $95 \%$ CI: 14.1-26.1), or junior secondary school (10.2\%; 95\% CI: $6.49-15.8)$, and secondary or higher (9.7\%; 95\% CI: 4.94-18.3). Among those who were aware, $38.0 \%$ (95\% CI: 30.6-46.0) had an annual income of $\leq 10000 \mathrm{ZMW}$ and $36.4 \%$ (95\% CI: 27.8-45.9) had an annual income of $>20000 \mathrm{ZMW}$, while among those who were not aware, nearly half (48.0\%; 95\% CI: $40.1-56.0)$ and $18.6 \%$ (95\% CI: $12.7-26.3)$ had an annual income of $\leq 10000 \mathrm{ZMW}$ and $>20000 \mathrm{ZMW}$, respectively. About 14.6\% (95\% CI: 9.8-21.3) and almost 3 in 10 (28.6\%; 95\% CI: $22.3-35.9)$ of those who were aware and not aware, respectively, did not have information on income. The distributions of age and marital status did not differ significantly between those who were aware and were not aware of health warnings.

Among all adults who currently smoked, a higher percentage of adults who currently smoked who self-reported thinking about quitting because of health warnings on cigarette packages (from here on referred to as 'thinking about quitting') were male (96.2\%; 95\% CI: 92.6-98.1), 29.1\% (95\% CI: $21.1-$ $38.5)$ had completed primary school and $29.4 \%$ (95\% CI: 19.8-41.2) completed junior secondary school, with similar proportions of those who had income levels $\leq 10000 \mathrm{ZMW}$ (37.8\%; 95\% CI: 28.9-47.6), and $>20000 \mathrm{ZMW}(37.9 \%$; 95\% CI: 27.1-50.2). Among those who were not thinking about quitting, 89\% (95\% CI: 84.6-92.3) were male, half completed less than primary school (37.5\%; 95\% CI: 31.5-44.0) or had no formal schooling (12.1\%; 95\% CI: $8.25-$ 17.4 ), and $45 \%$ (95\% CI: $38.4-52.0$ ) had an income level $\leq 10000$ ZMW (Table 1 ).

When restricted to the subset of those with awareness of health warnings, the sociodemographic distribution among those thinking about quitting was similar to the distribution for those thinking about quitting among all adults who currently smoked with the exception of education level, marital status, and income level (Table 1). Approximately $29.1 \%$ (95\% CI: $21.1-38.5$ ) and $29.4 \%$ (95\% CI: 19.8-41.2) of those aware of health warning labels and thinking about quitting completed primary school and junior secondary school, respectively, and $37.8 \%$ (95\% CI: 28.9-47.6) and $37.9 \%$ (95\% CI: 27.1-50.2) had income levels $\leq 10000$ and $>20000$, respectively. Among those who were not thinking about quitting, $24.6 \%$ (95\% CI: $14.2-39.1$ ) had completed primary school while a quarter (25.6\%; 95\% CI: 16.2-38.0) had completed junior secondary school and another quarter $(25.0 \%$; 95\% CI: 14.4-39.8) had completed secondary high school or higher; $38.3 \%$ (95\% CI: 25.9-52.4) and $33.2 \%$ (95\% CI: 20.7-48.7) had $\leq 10000$ and $>20000$ 


\section{Research Paper}

Table 1. Distribution of selected sociodemographic characteristics, overall and by awareness of health warnings and thinking about quitting, among adults who currently smoked tobacco aged 18-69 years in Zambia, STEPS Survey 2017

\begin{tabular}{|c|c|c|c|c|c|c|c|c|c|c|c|c|c|c|c|c|}
\hline \multirow[t]{3}{*}{ Characteristics } & \multirow{2}{*}{\multicolumn{4}{|c|}{ Overall }} & \multicolumn{4}{|c|}{$\begin{array}{c}\text { Aware of health warnings on cigarette } \\
\text { packages }\end{array}$} & \multicolumn{4}{|c|}{$\begin{array}{l}\text { Thinking about quitting because of } \\
\text { health warnings on cigarette packages } \\
\text { among adults who currently smoked }\end{array}$} & \multicolumn{4}{|c|}{$\begin{array}{c}\text { Thinking about quitting because of } \\
\text { health warnings on cigarette packages } \\
\text { among those aware of health warning } \\
\text { labels }\end{array}$} \\
\hline & & & & & \multicolumn{2}{|r|}{ Yes } & \multicolumn{2}{|r|}{ No } & \multicolumn{2}{|r|}{ Yes } & \multicolumn{2}{|r|}{ No } & \multicolumn{2}{|r|}{ Yes } & \multicolumn{2}{|r|}{ No } \\
\hline & $\begin{array}{c}\text { Unweighted } \\
n\end{array}$ & $\begin{array}{l}\text { Weighted } \\
n\end{array}$ & $\%$ & $95 \%$ CI & $\%$ & $95 \%$ CI & $\%$ & $95 \%$ CI & $\%$ & $95 \% \mathrm{CI}$ & $\%$ & $95 \%$ CI & $\%$ & $95 \%$ CI & $\%$ & $95 \%$ CI \\
\hline \multicolumn{17}{|l|}{ Age (years) } \\
\hline $18-29$ & 130 & 351661 & 38.4 & $32.2-44.9$ & 39.1 & $30.2-48.8$ & 37.5 & $29.7-45.9$ & 41.1 & $29.9-53.4$ & 36.7 & $30.0-44.1$ & 41.1 & $29.9-53.4$ & 35.0 & $22.5-49.9$ \\
\hline $30-44$ & 175 & 334976 & 36.6 & $31.4-42.0$ & 40.2 & $32.5-48.4$ & 32.0 & $25.6-39.1$ & 40.9 & $30.9-51.8$ & 33.9 & $28.3-40.1$ & 40.9 & $30.9-51.8$ & 38.7 & $28.2-50.3$ \\
\hline $45-59$ & 120 & 169037 & 18.5 & $15.0-22.4$ & 15.4 & $11.1-20.9$ & 22.4 & $17.0-28.9$ & 12.8 & $8.60-18.7$ & 21.8 & $17.2-27.2$ & 12.8 & 8.6-18.7 & 20.5 & $12.5-31.8$ \\
\hline $60-69$ & 50 & 60508 & 6.6 & $4.6-9.5$ & 5.4 & $2.80-10.0$ & 8.2 & $5.2-12.6$ & 5.1 & $2.31-10.9$ & 7.5 & $4.88-11.3$ & $\sim$ & & $\sim$ & \\
\hline \multicolumn{17}{|l|}{$\operatorname{Sex}^{\mathrm{a}, \mathrm{b}}$} \\
\hline Male & 414 & 840219 & 91.7 & 88.7-93.9 & 94.6 & $91.1-96.7$ & 88.1 & $83.2-91.7$ & 96.2 & $92.6-98.1$ & 89.0 & $84.6-92.3$ & 96.2 & $92.6-98.1$ & 91.3 & $82.2-95.9$ \\
\hline Female & 61 & 75963 & 8.3 & $6.1-11.3$ & 5.4 & $3.26-8.88$ & 11.9 & $8.35-16.8$ & 3.8 & $1.90-7.39$ & 11.0 & $7.73-15.4$ & $\sim$ & & $\sim$ & \\
\hline Bemba & 160 & 311684 & 34.0 & $27.3-41.5$ & 35.6 & $26.6-45.9$ & 32.0 & $24.2-40.9$ & 36.1 & $24.9-49.1$ & 32.8 & $25.7-40.7$ & 36.1 & $24.9-49.1$ & 34.7 & $21.9-50.1$ \\
\hline Chewa & 39 & 74285 & 8.1 & $5.3-12.2$ & 11.1 & $7.07-17.0$ & 4.3 & $1.9-9.3$ & 12.7 & $7.7-20.1$ & 5.4 & $2.89-9.82$ & 12.7 & 7.7-20.1 & 7.9 & $2.9-19.8$ \\
\hline Tonga & 38 & 66618 & 7.3 & $4.9-10.7$ & 6.1 & $3.24-11.0$ & 8.8 & $5.3-14.4$ & 6.0 & $2.7-12.7$ & 8.0 & $5.09-12.4$ & $\sim$ & & $\sim$ & \\
\hline Other & 237 & 461230 & 50.3 & $43.2-57.4$ & 46.8 & $37.3-56.5$ & 54.9 & $45.9-63.5$ & 44.6 & $32.9-56.9$ & 53.8 & $46.0-61.4$ & 44.6 & $32.9-56.9$ & 51.2 & $37.2-65.0$ \\
\hline Missing/refused & 1 & 2366 & 0.3 & $0.04-1.8$ & $\sim$ & & $\sim$ & & $\sim$ & & $\sim$ & & $\sim$ & & $\sim$ & \\
\hline \multicolumn{17}{|l|}{ Education level ${ }^{\mathrm{a}, \mathrm{b}}$} \\
\hline No formal schooling & 48 & 77953 & 8.5 & $6.0-12.0$ & 2.3 & $1.13-4.43$ & 16.5 & $11.1-23.7$ & 2.5 & $1.1-5.7$ & 12.1 & $8.25-17.4$ & $\sim$ & & $\sim$ & \\
\hline Less than primary school & 154 & 265145 & 28.9 & $24.2-34.2$ & 17.0 & $12.0-23.5$ & 44.1 & $36.7-51.8$ & 14.6 & $9.3-22.4$ & 37.5 & $31.5-44.0$ & 14.6 & $9.3-22.4$ & 21.7 & $12.7-34.7$ \\
\hline Primary school completed & 116 & 219896 & 24.0 & $19.6-29.1$ & 27.6 & $21.1-35.2$ & 19.4 & $14.1-26.1$ & 29.1 & $21.1-38.5$ & 21.0 & $15.8-27.2$ & 29.1 & $21.1-38.5$ & 24.6 & $14.2-39.1$ \\
\hline $\begin{array}{l}\text { Junior secondary school } \\
\text { completed }\end{array}$ & 88 & 185605 & 20.3 & $15.4-26.1$ & 28.1 & $20.6-37.2$ & 10.2 & $6.49-15.8$ & 29.4 & $19.8-41.2$ & 14.8 & $10.7-20.1$ & 29.4 & $19.8-41.2$ & 25.6 & $16.2-38.0$ \\
\hline $\begin{array}{l}\text { Secondary high school } \\
\text { completed or higher }\end{array}$ & 68 & 165300 & 18.0 & $13.8-23.3$ & 24.6 & $18.3-32.2$ & 9.7 & $4.94-18.3$ & 24.4 & $17.5-32.9$ & 14.3 & $9.38-21.1$ & 24.4 & $17.5-32.9$ & 25.0 & $14.4-39.8$ \\
\hline Missing/refused & 1 & 2284 & 0.3 & $0.03-1.8$ & $\sim$ & & $\sim$ & & $\sim$ & & $\sim$ & & $\sim$ & & $\sim$ & \\
\hline
\end{tabular}




\section{Research Paper}

Table 1. Continued

\begin{tabular}{|c|c|c|c|c|c|c|c|c|c|c|c|c|c|c|c|c|}
\hline \multirow[t]{3}{*}{ Characteristics } & \multirow{2}{*}{\multicolumn{4}{|c|}{ Overall }} & \multicolumn{4}{|c|}{$\begin{array}{c}\text { Aware of health warnings on cigarette } \\
\text { packages }\end{array}$} & \multicolumn{4}{|c|}{$\begin{array}{l}\text { Thinking about quitting because of } \\
\text { health warnings on cigarette packages } \\
\text { among adults who currently smoked }\end{array}$} & \multicolumn{4}{|c|}{$\begin{array}{c}\text { Thinking about quitting because of } \\
\text { health warnings on cigarette packages } \\
\text { among those aware of health warning } \\
\text { labels }\end{array}$} \\
\hline & & & & & \multicolumn{2}{|r|}{ Yes } & \multicolumn{2}{|r|}{ No } & \multicolumn{2}{|r|}{ Yes } & \multicolumn{2}{|r|}{ No } & \multicolumn{2}{|r|}{ Yes } & \multicolumn{2}{|r|}{ No } \\
\hline & $\begin{array}{c}\text { Unweighted } \\
n\end{array}$ & $\begin{array}{c}\text { Weighted } \\
n\end{array}$ & $\%$ & $95 \% \mathrm{CI}$ & $\%$ & $95 \% \mathrm{CI}$ & $\%$ & $95 \%$ CI & $\%$ & $95 \% \mathrm{CI}$ & $\%$ & $95 \% C I$ & $\%$ & $95 \% C I$ & $\%$ & $95 \% \mathrm{CI}$ \\
\hline \multicolumn{17}{|l|}{ Marital status } \\
\hline Currently married & 326 & 618673 & 67.5 & $61.1-73.3$ & 62.8 & $53.6-71.2$ & 73.6 & $65.6-80.2$ & 65.4 & $54.1-75.2$ & 68.8 & $61.4-75.4$ & 65.4 & $54.1-75.2$ & 57.5 & $42.7-71.0$ \\
\hline Not married & 149 & 297509 & 32.5 & $26.7-38.9$ & 37.2 & $28.8-46.4$ & 26.4 & $19.8-34.4$ & 34.6 & $24.8-45.9$ & 31.2 & $24.6-38.6$ & 34.6 & $24.8-45.9$ & 42.5 & $29.0-57.3$ \\
\hline \multicolumn{17}{|l|}{ Income level ${ }^{\mathrm{a}, \mathrm{b}}$ (ZMW) } \\
\hline$\leq 10000$ & 207 & 388352 & 42.4 & $36.7-48.3$ & 38.0 & $30.6-46.0$ & 48.0 & $40.1-56.0$ & 37.8 & $28.9-47.6$ & 45.1 & $38.4-52.0$ & 37.8 & $28.9-47.6$ & 38.3 & $25.9-52.4$ \\
\hline$>10000$ and $\leq 20000$ & 42 & 76062 & 8.3 & $5.9-11.6$ & 11.0 & $7.4-16.3$ & 4.8 & $2.42-9.40$ & 11.8 & $7.1-19.0$ & 6.2 & $3.77-10.0$ & 11.8 & $7.1-19.0$ & 9.4 & $4.7-18.1$ \\
\hline$>20000$ & 112 & 261343 & 28.5 & $22.7-35.2$ & 36.4 & $27.8-45.9$ & 18.6 & $12.7-26.3$ & 37.9 & $27.1-50.2$ & 22.9 & $17.0-30.0$ & 37.9 & $27.1-50.2$ & 33.2 & $20.7-48.7$ \\
\hline Missing/don't know/refused & 114 & 190425 & 20.8 & $16.6-25.7$ & 14.6 & $9.8-21.3$ & 28.6 & $22.3-35.9$ & 12.4 & $7.3-20.4$ & 25.8 & $20.3-32.1$ & 12.4 & $7.3-20.4$ & 19.0 & $10.1-32.9$ \\
\hline
\end{tabular}

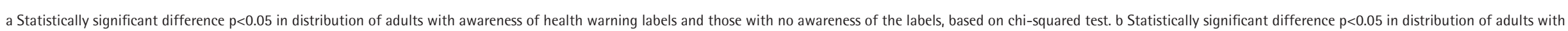

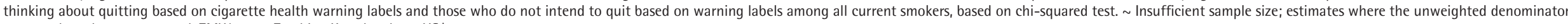
was $<25$ have been suppressed. ZMW: 1000 Zambian Kwacha about US $\$ 56$. 


\section{Research Paper}

Table 2. Prevalence ratios (PR) for the association of selected sociodemographic characteristics with awareness of health warnings and thinking about quitting among adults who currently smoked tobacco aged 18-69 years in Zambia, STEPS survey 2017

\begin{tabular}{|c|c|c|c|c|c|c|c|c|c|c|c|c|c|c|c|c|c|c|}
\hline \multirow[t]{2}{*}{ Characteristics } & \multicolumn{6}{|c|}{ Aware of health warnings on cigarette packages } & \multicolumn{6}{|c|}{$\begin{array}{l}\text { Thinking about quitting because of health warnings on } \\
\text { cigarette packages among all adults who currently smoked }\end{array}$} & \multicolumn{6}{|c|}{$\begin{array}{c}\text { Thinking about quitting because of health warnings on } \\
\text { cigarette packages among those aware of health warning } \\
\text { labels }\end{array}$} \\
\hline & $\%$ & $95 \%$ CI & $P R$ & $95 \%$ CI & $A P R$ & $95 \% \mathrm{CI}$ & $\%$ & $95 \% \mathrm{CI}$ & $P R$ & $95 \% \mathrm{CI}$ & APR & $95 \%$ CI & $\%$ & $95 \%$ CI & $P R$ & $95 \% \mathrm{CI}$ & $A P R$ & $95 \% \mathrm{CI}$ \\
\hline \multicolumn{19}{|l|}{ Age (years) } \\
\hline 18-29 (Ref.) & 57.0 & $45.1-68.1$ & 1 & & 1 & & 40.1 & $28.6-52.9$ & 1 & & 1 & & 70.4 & $55.0-82.3$ & 1 & & 1 & \\
\hline $30-44$ & 61.5 & $52.8-69.5$ & 1.08 & $0.85-1.36$ & 1.26 & $1.02-1.54^{\mathrm{a}}$ & 41.9 & $33.4-51.0$ & 1.04 & $0.72-1.52$ & 1.11 & $0.79-1.56$ & 68.2 & $57.2-77.4$ & 0.97 & $0.75-1.24$ & 0.86 & $0.66-1.11$ \\
\hline $45-59$ & 46.6 & $36.1-57.4$ & 0.82 & $0.60-1.11$ & 1.06 & $0.81-1.38$ & 26.0 & $18.3-35.6$ & 0.65 & $0.41-1.02$ & 0.78 & $0.50-1.20$ & 55.8 & $39.5-71.0$ & 0.79 & $0.56-1.13$ & 0.72 & $0.50-1.04$ \\
\hline $60-69$ & 45.5 & $27.4-64.9$ & 0.80 & $0.49-1.29$ & 1.19 & $0.85-1.65$ & 29.0 & $14.1-50.4$ & 0.72 & $0.36-1.45$ & 1.03 & $0.62-1.72$ & $\sim$ & & $\sim$ & & $\sim$ & \\
\hline \multicolumn{19}{|l|}{ Sex } \\
\hline Male (Ref.) & 57.7 & $51.0-64.2$ & 1 & & 1 & & 39.3 & $32.8-46.2$ & 1 & & 1 & & 68.1 & $59.5-75.6$ & 1 & & 1 & \\
\hline Female & 36.6 & $24.9-50.1$ & 0.63 & $0.44-0.92$ & 0.78 & $0.60-1.01$ & 17.1 & $8.70-30.8$ & 0.43 & $0.23-0.84^{b}$ & 0.51 & $0.27-0.96^{b}$ & $\sim$ & & $\sim$ & & $\sim$ & \\
\hline \multicolumn{19}{|l|}{ Ethnicity } \\
\hline Bemba (Ref.) & 58.6 & $48.1-68.4$ & 1 & & 1 & & 39.7 & $28.8-51.8$ & 1 & & 1 & & 67.8 & $51.7-80.5$ & 1 & & 1 & \\
\hline Chewa & 76.5 & $59.3-87.9$ & 1.31 & $1.01-1.68^{b}$ & 1.43 & $1.17-1.74^{b}$ & 58.4 & $41.7-73.5$ & 1.47 & $0.98-2.22$ & 1.55 & $1.03-2.35^{b}$ & 76.4 & $51.8-90.7$ & 1.13 & $0.80-1.59$ & 1.11 & $0.77-1.60$ \\
\hline Other & 52.0 & $42.7-61.1$ & 0.89 & $0.69-1.14$ & 1 & $0.81-1.22$ & 33.1 & $24.8-42.7$ & 0.83 & $0.56-1.25$ & 0.96 & $0.68-1.37$ & 63.8 & $51.6-74.4$ & 0.94 & $0.71-1.25$ & 0.97 & $0.74-1.27$ \\
\hline \multicolumn{19}{|l|}{$\begin{array}{l}\text { Education } \\
\text { level }\end{array}$} \\
\hline $\begin{array}{l}\text { No formal } \\
\text { schooling }\end{array}$ & 14.8 & $7.4-27.4$ & 0.52 & $0.26-1.03$ & 0.41 & $0.20-0.82^{b}$ & 11.2 & $5.0-23.1$ & 0.59 & $0.25-1.41$ & 0.56 & $0.23-1.37$ & $\sim$ & & $\sim$ & & $\sim$ & \\
\hline $\begin{array}{l}\text { Less than } \\
\text { primary } \\
\text { school (Ref.) }\end{array}$ & 32.9 & $24.4-42.6$ & 1 & & 1 & & 18.9 & $12.6-27.5$ & 1 & & 1 & & 57.6 & $39.0-74.3$ & 1 & & 1 & \\
\hline $\begin{array}{l}\text { Primary } \\
\text { school } \\
\text { completed }\end{array}$ & 64.3 & $53.1-74.2$ & 1.89 & $1.38-2.58^{b}$ & 1.77 & $1.31-2.39^{b}$ & 45.3 & $34.0-57.2$ & 2.39 & $1.49-3.84^{b}$ & 2.27 & $1.43-3.62^{b}$ & 70.5 & $54.3-82.8$ & 1.22 & $0.83-1.79$ & 1.27 & $0.87-1.85$ \\
\hline $\begin{array}{l}\text { Junior } \\
\text { secondary } \\
\text { school } \\
\text { completed }\end{array}$ & 77.8 & $65.5-86.6$ & 2.17 & $1.61-2.93^{b}$ & 2.10 & $1.57-2.79^{b}$ & 54.3 & $39.6-68.4$ & 2.87 & $1.78-4.64^{b}$ & 2.76 & $1.72-4.41^{b}$ & 69.9 & $54.3-82.0$ & 1.21 & $0.84-1.76$ & 1.21 & $0.84-1.74$ \\
\hline
\end{tabular}




\section{Research Paper}

Table 2. continued

\begin{tabular}{|c|c|c|c|c|c|c|c|c|c|c|c|c|c|c|c|c|c|c|}
\hline \multirow[t]{2}{*}{ Characteristics } & \multicolumn{6}{|c|}{ Aware of health warnings on cigarette packages } & \multicolumn{6}{|c|}{$\begin{array}{l}\text { Thinking about quitting because of health warnings on } \\
\text { cigarette packages among all adults who currently smoked }\end{array}$} & \multicolumn{6}{|c|}{$\begin{array}{c}\text { Thinking about quitting because of health warnings on } \\
\text { cigarette packages among those aware of health warning } \\
\text { labels }\end{array}$} \\
\hline & $\%$ & $95 \% \mathrm{CI}$ & $P R$ & $95 \% \mathrm{CI}$ & $A P R$ & $95 \% \mathrm{CI}$ & $\%$ & $95 \% \mathrm{CI}$ & $P R$ & $95 \% \mathrm{CI}$ & APR & $95 \% \mathrm{CI}$ & $\%$ & $95 \% \mathrm{CI}$ & $P R$ & $95 \% \mathrm{CI}$ & APR & $95 \%$ CI \\
\hline $\begin{array}{l}\text { Secondary } \\
\text { high school } \\
\text { completed or } \\
\text { higher }\end{array}$ & 76.2 & $58.7-87.8$ & 2.13 & $1.52-2.98^{b}$ & 1.98 & $1.41-2.78^{b}$ & 50.5 & $36.3-64.7$ & 2.67 & $1.66-4.30^{b}$ & 2.43 & $1.49-3.98^{b}$ & 66.3 & $49.9-79.5$ & 1.15 & $0.78-1.69$ & 1.18 & $0.80-1.73$ \\
\hline \multicolumn{19}{|l|}{$\begin{array}{l}\text { Marital } \\
\text { status }\end{array}$} \\
\hline $\begin{array}{l}\text { Currently } \\
\text { married (Ref.) }\end{array}$ & 52.0 & $44.9-59.1$ & 1 & & 1 & & 36.3 & $29.6-43.5$ & 1 & & 1 & & 69.7 & $59.9-78.0$ & 1 & & 1 & \\
\hline Not married & 64.1 & $52.9-74.0$ & 1.23 & $1.00-1.52^{b}$ & 1.17 & $0.96-1.43$ & 39.9 & $28.7-52.2$ & 1.10 & $0.78-1.55$ & 0.98 & $0.70-1.39$ & 62.2 & $47.2-75.2$ & 0.89 & $0.69-1.16$ & 0.81 & $0.60-1.08$ \\
\hline
\end{tabular}

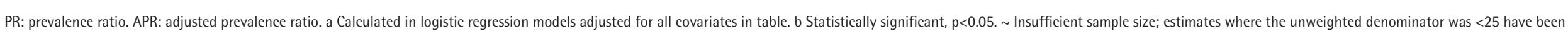
suppressed. 
ZMW income levels, respectively. Almost two-thirds (65.4\%; 95\% CI: 54.1-75.2) of those who were thinking about quitting were married compared to $57.5 \%$ (95\% CI: 42.7-71.0) who were not thinking about quitting.

\section{Prevalence and associated sociodemographic characteristics of awareness of health warnings and thinking about quitting}

The prevalence of awareness of health warnings among adults who currently smoked was relatively high among the 30-44 years age group (61.5\%; 95\% CI: 52.8-69.5), men (57.7\%; 95\% CI: 51.0-64.2), those of Chewa ethnicity (76.5\%; 95\% CI: 59.387.9), those who had completed junior secondary (77.8\%; 95\% CI: 65.5-86.6) or secondary high school (76.2\%; 95\% CI: 58.7-87.8), and those who were not married (64.1\%; 95\% CI: 52.9-74.0) (Table 2). In the unadjusted model, those of Chewa ethnicity were 1.31 times (95\% CI for PR: 1.01-1.68) more likely to be aware of health warnings compared with those of Bemba ethnicity. Those who had no formal schooling were 0.52 times (95\% CI: 0.26-1.03) less likely to be aware than those with less than primary school. Those who had completed primary school ( $\mathrm{PR}=1.89$; 95\% CI: 1.38-2.58), junior secondary school (PR=2.17; 95\% CI: 1.61-2.93) or secondary high school ( $\mathrm{PR}=2.13 ; 95 \%$ CI: $1.52-2.98)$ were more likely to be aware than those with less than primary schooling. Those who were not married were 1.23 times (95\% CI for PR: 1.00-1.52) more likely to be aware of health warnings compared to those who were married. Women ( $\mathrm{PR}=0.63$; 95\% CI: 0.44-0.92) were less likely to be aware of health warnings compared to men. In the adjusted model, being in the 30-44 age group ( $\mathrm{APR}=1.26 ; 95 \% \mathrm{CI}: 1.02-1.54)$, Chewa ethnicity $(\mathrm{APR}=1.43 ; 95 \% \mathrm{CI}: 1.17-1.74)$ and education: without formal schooling (APR $=0.41 ; 95 \%$ CI: $0.20-0.82)$, primary school (APR $=1.77 ; 95 \%$ CI: $1.31-2.93)$, junior secondary school (APR $=2.10 ; 95 \%$ CI: 1.57-2.79) and secondary high school $(\mathrm{APR}=1.98$; 95\% CI: 1.41-2.78) remained significantly more or less likely to be aware of health warnings. Sex and marital status were no longer significant after adjusting for other sociodemographic variables.

Among all adults who currently smoked, thinking about quitting had similar findings to those of awareness of health warnings (Table 2). The prevalence was relatively high among those aged $18-29$ and $30-44$ years $(40.1 \%$; 95\% CI: $28.6-$ 52.9, and 41.9\%; 95\% CI: 33.4-51.0, respectively), men (39.3\%; 95\% CI: 32.8-46.2), those of Chewa ethnicity (58.4\%; 95\% CI: 41.7-73.5), those who had completed junior secondary and secondary high school (54.3\%; 95\% CI: 39.6-68.4, and 50.5\%; 95\% CI: 36.3-64.7, respectively) and those who were not married (39.9\%; 95\% CI: 28.7-52.2). In the unadjusted model, those with any formal education (PR range: 2.39; 95\% CI: 1.49-3.84 [primary school completed] to 2.67 ; $95 \%$ CI: $1.66-4.30$ [secondary high school completed]) were more likely to report thinking about quitting than those with no formal schooling ( $\mathrm{PR}=0.59$; 95 CI: 0.25-1.41). Women $(\mathrm{PR}=0.43$; 95\% CI: $0.23-0.84)$ were less likely to report thinking about quitting than men. In the adjusted model, sex and education level remained significant; additionally, those of Chewa ethnicity $(\mathrm{APR}=1.55 ; 95 \% \mathrm{CI}: 1.03-2.35)$ were more likely to report thinking about quitting than those of Bemba ethnicity.

When restricted to current smokers who were aware of health warning labels, the prevalence of thinking about quitting was $70.4 \%$ (95\% CI: 55.0-82.3) among those aged 18-29 years, $76.4 \%$ (95\% CI: 51.8-90.7) among those of Chewa ethnicity, $70.5 \%$ (95\% CI: 54.3-82.8) and 69.9\% (95\% CI: 54.3-82.0), respectively, among those who had completed primary and junior secondary schools, and 69.7\% (95\% CI: 59.9-78.0) among those who were married (Table 2). There were no sociodemographic characteristics that were significantly associated with thinking about quitting in unadjusted or adjusted models.

\section{DISCUSSION}

Our analyses found that among adults who currently smoke, sociodemographic differences exist in awareness of health warning labels on cigarette packages, particularly in sex, ethnicity, and education level, but no sociodemographic differences were found in thinking about quitting among tobacco smokers aware of the health warnings on cigarette packages. Generally, those who had completed primary school or above were more likely to be aware of health warnings, with the magnitude of the association increasing with higher education level. Findings from 
this study may highlight the need for clear and easy to understand pictorial health warnings on cigarette packages that could be understood by all adults of any level of education, sex, or ethnicity in Zambia.

We found that certain sociodemographic characteristics were significantly associated with awareness of health warnings and thinking about quitting among all adults who currently smoked. Even though the proportion of female current tobacco smokers in Zambia was relatively low, our finding that women were less likely to notice health warnings and think about quitting than men is like that reported in some Asian countries such as Vietnam, Malaysia and Thailand ${ }^{20,21,26,30-32}$. Women, especially those of low socioeconomic status, may be generally less responsive to tobacco control interventions than men not only because fewer women attain higher levels of education compared to men in $\mathrm{Zambia}^{7}$, but there has also been limited integration of gender considerations into multiple elements of tobacco control policies, including messages on the harms of tobacco use $\mathrm{u}^{33-35}$. This may, in part, explain our findings that suggest that women may be less likely to have awareness of health warning labels or thinking about quitting. We also found that those of Chewa ethnicity were more likely to be aware of health warnings and thinking about quitting than were those of Bemba ethnicity. Most adults of Chewa ethnicity reside in the Eastern Province, where smoking prevalence is one of the highest in Zambia ${ }^{32}$ and tobacco farming is common ${ }^{36}$; however, there is very little research on differences in tobacco use, awareness of health warnings, and thinking about quitting across ethnic groups, particularly in Zambia. While more research is required to understand these findings, it is important to ensure tobacco control interventions and campaigns are implemented equitably across sex and ethnic groups.

Previous studies have shown that smoking prevalence is highest among those with lower education level, decreasing as the level of education increases ${ }^{7,25,32}$. Not only are those with less education more likely to smoke, but they are also less likely to be aware of health warnings ${ }^{7,25,32,37}$, a finding like those observed in our study. We observed low levels of awareness of health warnings among those with no formal schooling (14.8\%); those who had some levels of education were 1.89 to 2.13 times as likely to be aware of health warnings, as opposed to those who had no formal schooling. A study conducted in 2014 showed that $40.0 \%$ of Zambian adults who currently smoked could not read the health warning at all, and another $14.0 \%$ read it with difficulty ${ }^{23}$. Several studies, including those from the United States and Europe, have concluded that people with less education were more responsive to graphic health warnings or perceived them as more effective than text-only warnings ${ }^{16,19,21,25,38}$. Furthermore, Zambian adults who currently smoked were reported to be less likely to be aware of several important smokingrelated health effects such as lung cancer, heart disease, and stroke compared to smokers in other countries ${ }^{23}$.

We found similar patterns in the associations between sociodemographic characteristics and thinking about quitting as we did for awareness of health warnings; however, in our model that included only those who were already aware of health warnings, these associations were no longer observed. This could be due, in part, to the small sample size observed in our study. Alternatively, it could possibly demonstrate that, while there are disparities in awareness across sociodemographic groups, warning labels are similarly effective at causing adults who smoke to think about quitting once they are aware of warning labels ${ }^{25,39-42}$. While results varied by sociodemographic group, over half of those who were aware of health warnings also indicated thinking about quitting, highlighting the importance of increasing awareness. This may present an opportunity for using health warnings as a means of educating smokers about the harms of smoking with messages such as 'smoking causes lung cancer' or 'smoking can lead to gangrene' or implementing pictorial health warnings ${ }^{15,23,43}$. Article 11 of the WHO Framework Convention on Tobacco Control (FCTC) requires the adoption of health warnings on tobacco packages and recommends, in part, that the warnings are large, clear, visible, legible and include pictures to enhance their effectiveness ${ }^{8}$.

Several studies from developed countries such as the USA, Japan, United Kingdom, Canada and Australia have reported that large text and pictorial health warnings are associated with an increased likelihood of quitting as opposed to single text- 
only warnings, regardless of the age or education

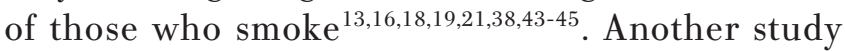
showed that while effectiveness of health warnings on cigarette packages varied, pictorial warnings with personal testimonials were the most effective across more than 100 countries $^{41}$. Pictorial warnings have several advantages relative to text-only warnings, including ability to attract and hold attention, elicit more negative smoking attitudes, and increase thinking about quitting ${ }^{37,46}$. Some Sub-Saharan African countries such as Uganda and Mauritius have adopted pictorial health warnings ${ }^{14}$. For example, Mauritius adopted large pictorial warning labels covering over $60-70 \%$ of cigarette packages, which resulted in increased awareness of health warnings from $58.0 \%$ to $83.4 \%$ over the course of two years post-implementation ${ }^{15}$. Zambia may have a similar opportunity to increase the awareness of health warnings and thinking about quitting among those who smoke and deter initiation among those who do not smoke. Implementing the WHOFCTC recommendation of large and pictorial health warnings may mitigate the sociodemographic differences related to awareness and thinking about quitting.

\section{Limitations}

This study has several limitations. First, this was a secondary analysis of a cross-sectional survey so causality cannot be determined between sociodemographic factors and awareness of health warnings and thinking about quitting. Second, selfreport responses could be subject to recall or social desirability biases, leading to underreporting of smoking or overreporting of thinking about quitting (e.g. some participants may intend to quit smoking for reasons other than noticing health warnings and thus would not be captured), although several studies have reported a high correlation between self-reports and biomarkers of smoking ${ }^{47-49}$. Third, we were not able to measure important variables with STEPS data such as language, region, or knowledge of health harms of tobacco, therefore we were unable to account for these factors in our analyses. For example, although English is the official language in Zambia, it is not the first language for most Zambians. Evidence suggests that about $69 \%$ of cigarette packages in Zambia carry a single text-only warning in English, 14\% are in another foreign language, and 18\% have no visible warnings ${ }^{23}$, underscoring the significant role language could play in responsiveness to health warnings. Additionally, we opted not to include income in our models due to a high proportion of missing data; thus, were unable to assess any associations between household income with awareness of health warnings and/or thinking about quitting. Despite these limitations, our study is unique in that, to our knowledge, this is the first time a study describing sociodemographic characteristics associated with awareness of health warnings and thinking about quitting has been conducted in Zambia. Another strength of this study lies in the ability to generalize its findings as it was based on the nationally representative 2017 Zambia STEPs survey.

\section{CONCLUSIONS}

Sociodemographic characteristics such as sex, ethnicity, and education level were significantly associated with awareness of cigarette health warnings. Among cigarette smokers aware of health warnings, no sociodemographic differences in thinking about quitting were found. Findings from this study indicated that current tobacco smokers who had primary school or above education were more likely to be aware of health warnings, which suggests the need for clear, easy to understand, pictorial health warnings on cigarette packages in Zambia. Implementation of stronger policies requiring health warnings with these characteristics may increase awareness of health warnings and thinking about quitting smoking, particularly among those with lower levels of education. Additionally, while a small percentage of women currently smoke tobacco in Zambia, there may be a need for targeted campaigns among women as they were less likely to be aware of health warnings and thinking about quitting. Equitable implementation of tobacco control campaigns may need to target regions or ethnicities (e.g. Chewa) in which smoking prevalence is higher compared to other regions of the country.

\section{REFERENCES}

1. World Health Organization. WHO report on the global tobacco epidemic 2019: offer help to quit tobacco use. July 25, 2019. Accessed December 10, 2021. https:// www.who.int/publications/i/item/9789241516204

2. Zambia- Country Profile. Tobacco Tactics. September 13, 2021. Accessed December 10, 2021. https:// 
tobaccotactics.org/wiki/zambia-country-profile/

3. Republic of Zambia, Ministry of Health. Zambia STEPS for non-communicable diseases risk factors. 2017. Accessed December 10, 2021. https://www.who.int/ ncds/surveillance/steps/Zambia-NCD-STEPS-SurveyReport-2017.pdf?ua=1

4. Central Statistical Office, Central Board of Health. Zambia Demographic and Health Survey 2001-2002. The World Bank. June 6, 2017. Accessed December 10, 2021. https://microdata.worldbank.org/index.php/ catalog/1526

5. Central Statistical Office. Zambia Demographic and Health Survey 2007. The World Bank. June 16, 2017. Accessed December 10, 2021. https://microdata.worldbank.org/ index.php/catalog/1527

6. Central Statistical Office, Ministry of Health. Zambia Demographic and Health Survey 2013-14. The World Bank. June 16, 2017. Accessed December 10, 2021. https://microdata.worldbank.org/index.php/ catalog/2246

7. Zambia Statistics Agency, Ministry of Health. Zambia Demographic and Health Survey 2018. The World Bank. February 25, 2020. Accessed December 10, 2021. https:// microdata.worldbank.org/index.php/catalog/3597

8. World Health Organization. WHO Framework Convention on Tobacco Control. World Health Organization; 2003. Updated 2005. Accessed December 10, 2021. http://apps.who.int/iris/bitstream/ handle/10665/42811/9241591013.pdf;jsessionid= C2DC7397764BE889780DB25F261442EF? sequence $=1$

9. World Health Organization Regional Office for Africa. The WHO framework convention on tobacco control: 10 years of implementation in the African region. World Health Organization; 2015. Accessed December 10, 2021. https://www.afro.who.int/sites/default/files/2017-06/ who-fctc-10-year_report_web.pdf

10. World Health Organization. Report card on the WHO Framework Convention on Tobacco Control Zambia. 2017. Accessed APRil 27, 2021. https://www.afro.who. int/sites/default/files/2017-09/Zambia_report_card_0. pdf

11. World Health Organization. MPOWER: A POLICY PACKAGE TO REVERSE THE TOBACCO EPIDEMIC. World Health Organization; 2008. Accessed December 10, 2021. https://apps.who.int/iris/bitstream/ handle/10665/43888/9789241596633_eng.pdf

12. Canadian Cancer Society. Cigarette package health warnings: international status report. Canadian Cancer Society. 5th ed. 2014. Accessed December 10, 2021. https://tobaccolabels.s3.ca-central-1.amazonaws.com/ uploads/2016/11/Cigarette-Package-Health-WarningsInternational-Status-Report-English-CCS-Oct-2016.pdf

13. Hnin TT, Shein NNN, Aye SKK. Awareness and Perceptions on Health Warning Labels on Cigarette Packs among Smokers: A Cross-Sectional Study. BioMed Res
Int. 2020;2020:9462903. doi:10.1155/2020/9462903

14. Centers for Disease Control and Prevention. The Global Tobacco Surveillance System (GTSS) African Region Atlas. 2018. Accessed December 10, 2021. https:// africanatlas.gtssacademy.org/

15. Green AC, Kaai SC, Fong GT, Driezen P, Quah AC, Burhoo P. Investigating the effectiveness of pictorial health warnings in Mauritius: findings from the ITC Mauritius survey. Nicotine Tob Res. 2014;16(9):12401247. doi:10.1093/ntr/ntu062

16. Chung-Hall J, Fong GT, Meng G, et al. Effectiveness of Text-Only Cigarette Health Warnings in Japan: Findings from the 2018 International Tobacco Control (ITC) Japan Survey. Int J Environ Res Public Health . 2020;17(3):952. doi:10.3390/ijerph17030952

17. Fathelrahman AI, Omar M, Awang R, Cummings KM, Borland R, Samin A. Impact of the new Malaysian cigarette pack warnings on smokers' awareness of health risks and interest in quitting smoking. Int J Environ Res Public Health. 2010;7(11):4089-4099. doi:10.3390/ijerph7114089

18. Hammond D, Fong GT, McNeill A, Borland R, Cummings KM. Effectiveness of cigarette warning labels in informing smokers about the risks of smoking: findings from the International Tobacco Control (ITC) Four Country Survey. Tob Control. 2006;15(Suppl 3):iii19-iii25. doi:10.1136/tc.2005.012294

19. Hitchman SC, Mons U, Nagelhout GE, et al. Effectiveness of the European Union text-only cigarette health warnings: findings from four countries. Eur J Public Health. 2012;22(5):693-699. doi:10.1093/eurpub/ckr099

20. Kaai SC, Fong GT, Goma F, et al. Identifying factors associated with quit intentions among smokers from two nationally representative samples in Africa: Findings from the ITC Kenya and Zambia Surveys. Prev Med Reports. 2019;15:100951. doi:10.1016/j.pmedr.2019.100951

21. Ngoc BN, Thu NT, Bao GK, et al. Salience and impact of health warning label on cigarette packs in Vietnam: findings from the Global Adult Tobacco Survey 2015. Behav Med. 2019;45(1):30-39. doi:10.1080/08964289.2018.1434117

22. Noar SM, Francis DB, Bridges C, Sontag JM, Ribisl KM, Brewer NT. The impact of strengthening cigarette pack warnings: Systematic review of longitudinal observational studies. Soc Sci Med. 2016;164:118-129. doi:10.1016/j.socscimed.2016.06.011

23. ITG Project. ITG Zambia Wave 2 (2014) Technical Report. 2015. Accessed December 10, 2021. https:// itcproject.s3.amazonaws.com/uploads/documents/ITC_ Zambia_W2_Technical_Report_FINAL_Sept10.pdf

24. Brown T, Platt S, Amos A. Equity impact of populationlevel interventions and policies to reduce smoking in adults: a systematic review. Drug Alcohol Depend. 2014;138:7-16. doi:10.1016/j.drugalcdep.2014.03.001

25. Shang C, Huang J, Cheng KW, He Y, Chaloupka FJ. 
The association between warning label requirements and cigarette smoking prevalence by education-findings from the Global Adult Tobacco Survey (GATS). Int J Environ Res Public Health. 2017;14(1):98. doi:10.3390/ijerph14010098

26. Salloum RG, Goma F, Chelwa G, et al. Cigarette price and other factors associated with brand choice and brand loyalty in Zambia: findings from the ITC Zambia Survey. Tob Control. 2015;24(Suppl 3):iii33-iii40. doi:10.1136/tobaccocontrol-2014-051878

27. Townsend L, Flisher AJ, Gilreath T, King G. A systematic literature review of tobacco use among adults 15 years and older in sub-Saharan Africa. Drug Alcohol Depend. 2006;84(1):14-27. doi:10.1016/j.drugalcdep.2005.12.008

28. Global Adult Tobacco Survey Collaborative Group. Global Adult Tobacco Survey (GATS): Fact Sheet Templates. Centers for Disease Control and Prevention; 2020. Accessed December 10, 2021. https://cdn.who. int/media/docs/default-source/ncds/ncd-surveillance/ gats/18_gats_analysispackage_final_23nov2 020 . pdf?sfvrsn=67e2065f_3

29. World Health Organization. Tobacco questions for surveys: a subset of key questions from the Global Adult Tobacco Survey (GATS): global tobacco surveillance system. 2nd ed. World Health Organization; 2011. Accessed December 10, 2021. https://apps.who.int/iris/ bitstream/handle/10665/87331/9789241500951_eng. pdf?sequence $=1 \&$ is Allowed $=y$

30. Ansara DL, Arnold F, Kishor S, Hsia J, Kaufmann R. Tobacco Use by Men and Women in 49 Countries with Demographic and Health Surveys. ICF International; 2013. DHS Comparative Reports No. 31. Accessed December 10, 2021. https://dhsprogram.com/pubs/pdf/ CR31/CR31.pdf

31. Nyirenda HT, Mulenga D, Silitongo M, Nyirenda HB, Nyirenda T. Spatial distribution and correlates of smoking in Zambia. BMJ Open. 2019;9(8):e030044. doi:10.1136/bmjopen-2019-030044

32. Sreeramareddy CT, Pradhan PM, Sin S. Prevalence, distribution, and social determinants of tobacco use in 30 sub-Saharan African countries. BMC Med. 2014;12(1):113. doi:10.1186/s12916-014-0243-x

33. Flor LS, Reitsma MB, Gupta V, Ng M, Gakidou E. The effects of tobacco control policies on global smoking prevalence. Nature Medicine. 2021;27(2):239-243. doi:10.1038/s41591-020-01210-8

34. Greaves L. The meanings of smoking to women and their implications for cessation. Int J Environ Res Public Health. 2015;12(2):1449-1465. doi:10.3390/ijerph120201449

35. Amos A, Greaves L, Nichter M, Bloch M. Women and tobacco: a call for including gender in tobacco control research, policy and practice. Tob Control. 2012;21(2):236243. doi:10.1136/tobaccocontrol-2011-050280
36. Labonté R, Lencucha R, Drope J, Packer C, Goma FM, Zulu R. The institutional context of tobacco production in Zambia. Global Health. 2018;14(1):1-12. doi:10.1186/s12992-018-0328-y

37. Noar SM, Hall MG, Francis DB, Ribisl KM, Pepper JK, Brewer NT. Pictorial cigarette pack warnings: a meta-analysis of experimental studies. Tob Control. 2016;25(3):341-354. doi:10.1136/tobaccocontrol-2014-051978

38. Cantrell J, Vallone DM, Thrasher JF, et al. Impact of tobacco-related health warning labels across socioeconomic, race and ethnic groups: results from a randomized web-based experiment. PloS One. 2013;8(1):e52206. doi:10.1371/journal.pone.0052206

39. Fathelrahman AI, Li L, Borland R, et al. Stronger pack warnings predict quitting more than weaker ones: finding from the ITC Malaysia and Thailand surveys. Tob Induced Dis. 2013;11(1):1-8. doi:10.1186/1617-9625-11-20

40. Reid JL, Mutti-Packer S, Gupta PC, et al. Influence of health warnings on beliefs about the health effects of cigarette smoking, in the context of an experimental study in four Asian countries. Int J Environ Res Public Health. 2017;14(8):868. doi:10.3390/ijerph14080868

41. Hammond D, Reid JL, Driezen P, et al. Are the same health warnings effective across different countries? An experimental study in seven countries. Nicotine Tob Res. 2019;21(7):887-895. doi:10.1093/ntr/nty248

42. Swayampakala K, Thrasher JF, Hammond D, et al. Pictorial health warning label content and smokers' understanding of smoking-related risks - a crosscountry comparison. Health Educ Res. 2015;30(1):35-45. doi:10.1093/her/cyu022

43. Tripathy JP, Verma M. Impact of Health Warning Labels on Cigarette Packs in India: Findings from the Global Adult Tobacco Survey 2016-17. Behav Med. 2020:1-10. doi:10.1080/08964289.2020.1796571

44. Hammond D. Health warning messages on tobacco products: a review. Tob Control. 2011;20(5):327-337. doi:10.1136/tc.2010.037630

45. Brewer NT, Parada Jr H, Hall MG, Boynton MH, Noar SM, Ribisl KM. Understanding why pictorial cigarette pack warnings increase quit attempts. Ann Behav Med. 2019;53(3):232-243. doi:10.1093/abm/kay032

46. National Center for Chronic Disease Prevention and Health Promotion (US) Office on Smoking and Health. Smoking Cessation: A Report of the Surgeon General [Internet]. US Department of Health and Human Services; 2020. Accessed December 10, 2021. https:// www.ncbi.nlm.nih.gov/books/NBK555591/

47. Wray JM, Gass JC, Miller EI, Wilkins DG, Rollins DE, Tiffany ST. A comparative evaluation of selfreport and biological measures of cigarette use in nondaily smokers. Psychol Assess. 2016;28(9):1043. doi:10.1037/pas0000227

48. Patrick DL, Cheadle A, Thompson DC, Diehr P, Koepsell T, Kinne S. The validity of self-reported smoking: a review 
and meta-analysis. Am J Public Health. 1994;84(7):10861093. doi:10.2105/AJPH.84.7.1086

49. Shiffman S, Dunbar MS, Benowitz NL. A comparison of nicotine biomarkers and smoking patterns in daily and nondaily smokers. Cancer Epidemiol Biomarkers Prev. 2014;23(7):1264-1272. doi:10.1158/1055-9965.EPI-131014

CONFLICTS OF INTEREST

The authors have completed and submitted the ICMJE Form for Disclosure of Potential Conflicts of Interest and none was reported.

\section{FUNDING}

This project was supported in part by an appointment to the Tobacco Scholars Program Fellowship administered by the CDC Foundation in partnership with the Centers for Disease Control and Prevention (CDC), and funded through a grant from the Bill \&t Melinda Gates Foundation.

\section{ETHICAL APPROVAL AND INFORMED CONSENT}

Ethical approval and informed consent were not required for this study, as existing data from the STEPS 2017 survey were used. The STEPS 2017 survey was approved by the University of Zambia Biomedical Research Ethics Committee.

DATA AVAILABILITY

Data sharing is not applicable to this article as no new data were created.

PROVENANCE AND PEER REVIEW

Not commissioned; externally peer reviewed. 